\title{
Crescimento da vasectomia no Sistema Único de Saúde entre 2009 a 2018: um estudo retrospectivo
}

\author{
The Vasectomy growth in the Government Health System between 2009 and 2018: a \\ retrospective study
}

Crecimiento de la vasectomía en el Sistema Único de Salud entre 2009 y 2018: uno estudio retrospectivo

Deivid Ramos dos Santos ${ }^{1 *}$, João Vitor da Costa Mangabeira ${ }^{1}$, Marcos Vinicius Amorim Silva ${ }^{1}$, Barbara Mendonça Paiva Antônio José ${ }^{1}$, Mateus Araújo Valente ${ }^{1}$, Paola dos Santos Dias ${ }^{1}$, Camila Beatriz da Silva Gomes ${ }^{1}$, Amanda Freitas Teixeira da Silva ${ }^{1}$, Luis Otávio Duarte Pinto ${ }^{1}$, Charles Alberto Villacorta de Barros ${ }^{1}$.

\section{RESUMO}

Objetivo: Descrever a epidemiologia da realização da vasectomia, estratificando-a por macrorregião brasileira entre 2009 a 2018. Métodos: Trata-se de uma pesquisa quantitativa, retrospectiva e descritiva, realizada a partir da coleta em base de dados disponibilizada pelo Sistema de Informações Hospitalares do departamento de informática do Sistema Único de Saúde do Brasil (SIH - SUS - DATASUS), referente ao procedimento de vasectomia, abrangendo uma série temporal de 2009 até 2018. Neste estudo foram incluídos todos os casos de pacientes submetidos a vasectomia do País. Resultados: Foram realizadas 271.142 vasectomias pela rede pública de saúde. A região Norte foi a que obteve o menor quantitativo no período estudado ( $n=5.914,2,18 \%)$. O Sudeste foi a região com o maior número $(n=152.715,56,32 \%)$. o Norte foi o que obteve maior crescimento, equivalente a aproximadamente $876 \%$. A região Sul possui a maior taxa de vasectomias por 100 mil habitantes, equivalendo a 193,26/100mil. O Norte e Nordeste tiveram as menores taxas, com 34,93/100 mil e 69,16/100 mil habitantes, respectivamente. Conclusão: Está havendo maior aceitação e procura da realização de vasectomia no Brasil confirmada pelo aumento do número de procedimentos notificados no Sistema Único de Saúde.

Palavras-chave: Vasectomia, Planejamento familiar, Saúde do homem.

\section{ABSTRACT}

Objective: Describe the vasectomy epidemiology, stratifying it by Brazilian macroregion from 2009 to 2018 . Methods: Quantitative, retrospective and descriptive research, conducted from the database collected by the Hospital Information System of the Informatics Department of the Brazilian Government Health System (SIHSUS - DATASUS), referring to the vasectomy procedure, covering a time series from 2009 to 2018 . This study included all cases of patients undergoing vasectomy in the country. Results: 271,142 vasectomies were performed by the public health network. The northern region was the one that obtained the lowest quantity in the studied period $(n=5,914,2.18 \%)$. The Southeast was the region with the largest number $(n=152,715$, $56.32 \%$ ). the North had the highest growth, equivalent to approximately $876 \%$. The southern region has the highest rate of vasectomies per 100,000 inhabitants, equivalent to $193,26 / 100,000$. The North and Northeast had the lowest rates, with 34.93 / 100 thousand and 69.16/100 thousand inhabitants, respectively. Conclusion: There is greater acceptance and demand for vasectomy in Brazil, confirmed by the increase in the number of procedures notified in the Government Health System.

Keywords: Vasectomy, Family planning, Men's health.

${ }^{1}$ Universidade do Estado do Pará (UEPa), Belém-Pará. *E-mail: deivid_ramos45@hotmail.com 


\section{RESUMEN}

Objetivo: Describer la epidemiología de la vasectomía, estratificándola por macroregión brasileña de 2009 a 2018. Métodos: nvestigación cuantitativa, retrospectiva y descriptiva, realizada a partir de la base de datos recopilada por el Sistema de Información Hospitalaria Sistema del Departamento de Informática del Sistema Único de Salud de Brasil (SIH-SUS - DATASUS), en referencia al procedimiento de vasectomía, que abarca una serie de tiempo de 2009 a 2018. Este estudio incluyó todos los casos de pacientes sometidos a vasectomía en el país. Resultados: La red de salud pública realizó 271.142 vasectomías. La región norte fue la que obtuvo la menor cantidad en el período estudiado $(n=5,914,2.18 \%)$. El sudeste fue la región con el mayor número ( $n=152,715,56.32 \%$ ). el norte tuvo el mayor crecimiento, equivalente a aproximadamente el $876 \%$. La región sur tiene la tasa más alta de vasectomías por cada 100,000 habitantes, equivalente a 193,26 / 100,000. El Norte y el Nordeste tuvieron las tasas más bajas, con 34.93 / 100 mil y 69.16/100 mil habitantes, respectivamente. Conclusión: Hay una mayor aceptación y demanda de la vasectomía en Brasil, confirmada por el aumento en el número de procedimientos notificados en el Sistema Único de Salud.

Palabras clave: Vasectomía, Planificación familiar, Salud masculina.

\section{INTRODUÇÃO}

Sabe-se que a vasectomia é um método de esterilização bastante efetivo (ROMERO PP et al., 2004) e consiste na obstrução cirúrgica dos ductos deferentes, vias responsáveis pelo transporte dos espermatozoides até o canal uretral, durante a ejaculação (EISENBERG ML e LIPSHILTZ LI, 2010). No mundo é estimado que aproximadamente 60 milhões de homens optam por esse procedimento (EISENBERG ML et al., 2009; OSIS MJD, et al., 2003).

Nota-se tendência à queda do número de filhos por casal em virtude do aumento dos métodos contraceptivos femininos e pela crescente busca de homens pela realização da vasectomia, sobretudo em países desenvolvidos e em metrópoles de países subdesenvolvidos (OSTROWSKI KA et al.,2018; VIEIRA EM, 1994).

Esse crescimento é explicado, em parte, porque a vasectomia possui menos complicações, retorno às atividades diárias, baixo custo e de fácil realização, quando comparada ao procedimento de esterilização feminina (TEMACH AJ, FEKUDA GA, ACHAMYELEH AA, 2017). Além disso, indicações médicas condicionadas ao risco para a mulher durante a gestação também levam pequena parcela masculina a optar pela vasectomia (EISENBERG ML, et al., 2009; OSTROWSKI KA, et al., 2018).

Outro fator adjuvante é que, recentemente, com a relativa melhora do serviço de planejamento familiar, foi possível canalizar esforços em novos grupos populacionais ainda não assistidos pelo Sistema Único de Saúde (SUS), como os homens, integrando-os aos serviços de planejamento familiar em saúde reprodutiva (EISENBERG ML e LIPSHILTZ LI, 2010; KAVANAUGH ML e ANDERSON RM, 2013; OSIS MJD, et al., 2003).

Em 1996, a Lei 9.263 foi publicada garantindo planejamento familiar como direito básico do cidadão e dever do Estado, caracterizando a vasectomia como método anticoncepcional reconhecido pelo Estado, devendo ser realizada por instituições públicas ou privadas, sob fiscalização do Sistema Único de Saúde (SCAFURI G,2002). Porém, sabe-se que essa não é a situação mais frequente no país e nem mesmo na nas regiões mais longínquas das áreas metropolitanas brasileiras, onde a disponibilidade e variedade de métodos contraceptivos ainda continuam a ser um problema para o acesso efetivo da população ao planejamento familiar (MARCHI NM, et al., 2011; OSIS MJD, et al., 2006).

No entanto, há trabalhos científicos mostrando demanda reprimida em centros de esterilização devido ao número insuficiente de cirurgias que podem ser realizadas semanalmente pelo SUS, principalmente, em relação à vasectomia e a lentidão para fazer os exames pré-operatórios quando encaminhar os pacientes para os serviços de referência credenciados (COSTA AM, et al., 2006; OSIS MJD, et al., 2009).

Além disso, a escassez de recursos humanos e físicos torna-se um fator que colabora para dificultar 0 acesso à vasectomia e aumenta a fila de espera, estimulando os homens a desistirem desse método contraceptivo e influenciando suas companheiras a optar pela laqueadura tubária, sendo esta mais onerosa 
ao SUS, com mais risco à mãe, porém menos demorada que a esterilização masculina indicando que a demanda recebida nos serviços públicos de saúde não conseguem resolução, implicando demora maior para obter a vasectomia do que a laqueadura. Isso inviabiliza a livre escolha e dos métodos anticoncepcionais e contribui para a esterilização tubária (OSIS MJD, et al., 2003; OSSIS MJD, et al., 2006).

Apesar de tais achados, ainda há poucos estudos epidemiológicos abordando a realização de vasectomia e sua reversão no Brasil, tanto por meio da rede pública de atenção à saúde quanto pela rede privada, mostrando um campo ainda pouco explorado num país continental e com diferenças regionais importantes relacionados ao acesso à saúde e aos serviços de planejamento familiar (PASQUALOTTO FF, et al., 2003; CHAILEE M e MICHELLE MI, 2015; CRISTOPHER EA e MOSHE W, 2009; PINTO LOAD, et al., 2019).

Assim o objetivo deste trabalho é descrever a epidemiologia da realização da vasectomia pela rede pública de saúde no Brasil, estratificando-a por macrorregião brasileira entre 2009 a 2018.

\section{MÉTODOS}

Este estudo é caracterizado como uma pesquisa quantitativa, de caráter retrospectivo e descritivo, realizado a partir da coleta de informações em base de dados disponibilizada pelo Sistema de Informações Hospitalares do departamento de informática do Sistema Único de Saúde do Brasil (SIH-SUS- DATASUS ), sob o código 0409040240, referente ao procedimento de vasectomia, abrangendo uma série temporal de 2009 até 2018. Neste estudo foram incluídos todos os casos de pacientes submetidos a vasectomia do País na atenção pública de saúde.

Os dados foram organizados e estratificados em tabelas e categorizados por estado, macrorregião e ano. Posteriormente, prosseguiu-se para a análise estatística com o programa Bioestat $\AA^{\circledR} 5.3$, por meio do teste Qui-quadrado, observando o p-valor < 0,05 e intervalo de confiança de 95\% para se rejeitar a hipótese de nulidade (EISENBERG ML e LIPSHULTZ LI, 2010).

Para a confecção do mapa de distribuição dos procedimentos por unidade da federação, utilizou-se o programa Tabwin $\AA 4.53$ disponibilizado pelo próprio Ministério da Saúde. Ressalta-se que não foi necessário submeter este trabalho ao Comitê de Ética em Pesquisa (CEP) por se tratar de dados secundários e disponibilizados em bando de domínio público.

\section{RESULTADOS}

Na Tabela 1 consta o número total de internações relacionados à realização de vasectomia pelo SUS. A região Norte foi a que obteve o menor quantitativo no período estudado $(n=5.914)$ sendo responsável por $2,18 \%$ dos casos. O Sudeste foi a região com o maior número $(n=152.715)$, correspondendo a $56,32 \%$ de todos os casos.

Tabela 1 - Número total de internações para o procedimento de vasectomia, distribuídos por ano e macrorregião do Brasil, entre 2009 a 2018.

\begin{tabular}{llllllllllll}
\hline Regiões & $\mathbf{2 0 0 9}$ & $\mathbf{2 0 1 0}$ & $\mathbf{2 0 1 1}$ & $\mathbf{2 0 1 2}$ & $\mathbf{2 0 1 3}$ & $\mathbf{2 0 1 4}$ & $\mathbf{2 0 1 5}$ & $\mathbf{2 0 1 6}$ & $\mathbf{2 0 1 7}$ & $\mathbf{2 0 1 8}$ & Total $^{*}$ \\
\hline Norte & 124 & 210 & 328 & 451 & 469 & 610 & 696 & 697 & 1.118 & 1.211 & 5.914 \\
Nordeste & 2.471 & 2.838 & 2.948 & 3.200 & 3.093 & 4.637 & 4.188 & 4.500 & 4.740 & 5.671 & 38.286 \\
Sudeste & 14.700 & 14.019 & 14.597 & 14.418 & 13.872 & 14.270 & 14.637 & 14.450 & 16.162 & 21.590 & 152.715 \\
Sul & 7.004 & 5.803 & 5.781 & 5.712 & 5.068 & 4.701 & 4.498 & 4.293 & 6.246 & 6.219 & 55.325 \\
Centro-Oeste & 2.012 & 1.751 & 1.968 & 1.695 & 1.487 & 1.432 & 1.686 & 2.139 & 2.459 & 2.273 & 18.902 \\
\hline Total & $\mathbf{2 6 . 3 1 1}$ & $\mathbf{2 4 . 6 2 1}$ & $\mathbf{2 5 . 6 2 2}$ & $\mathbf{2 5 . 4 7 6}$ & $\mathbf{2 3 . 9 8 9}$ & $\mathbf{2 5 . 6 5 0}$ & $\mathbf{2 5 . 7 0 5}$ & $\mathbf{2 6 . 0 7 9}$ & $\mathbf{3 0 . 7 2 5}$ & $\mathbf{3 6 . 9 6 4}$ & $\mathbf{2 7 1 . 1 4 2}$
\end{tabular}

Legenda: *Teste de tendência do $x^{2}(<0,001)$. Fonte: Ministério da Saúde - Sistema de Informações Hospitalares do SUS (SIH/SUS), 2019. 
Ainda conforme a tabela 1, observa-se aumento do número de vasectomias no país. Ao distribuir por macrorregião, o Norte foi o que obteve maior crescimento, equivalente a $876 \%$, seguido da região Nordeste com $129 \%$ e região Sul com $40 \%$, comparando o ano de 2009 com o ano de 2018 . A região Sudeste foi a única com crescimento negativo de $11 \%$. O crescimento total foi de $40,5 \%$, indo de 26.311 , em 2009 , para 36.964, em 2018.

A região Sul possui a maior taxa de vasectomias por 100 mil habitantes, equivalendo a 193,26/100mil, seguida da região Sudeste, com 181,77/100 mil, e Centro-Oeste com 126,10/100 mil. O Norte e Nordeste tiveram as menores taxas, com 34,93/100 mil e 69,16/100 mil habitantes, respectivamente (Tabela 2).

Tabela 2 - Número total de vasectomias por região, média ao ano, população residente média e distribuição de vasectomias por 100 mil habitantes entre 2009 a 2018.

\begin{tabular}{lllccc}
\hline Região & Número & Média anual & $\begin{array}{l}\text { Densidade } \\
\text { média }\end{array}$ & demográfica & $\begin{array}{l}\text { Taxa } \\
\text { hab* }^{*}\end{array}$ \\
\hline Norte & 5.914 & 591,4 & $16.933 .323,9$ & $\mathbf{1 0 0}$ mil \\
Nordeste & 38.286 & $3.828,6$ & $55.355 .019,0$ & 34,93 \\
Sudeste & 152.715 & $15.271,5$ & $84.015 .598,0$ & 69,16 \\
Sul & 55.325 & $5.532,5$ & $28.627 .882,3$ & 181,77 \\
Centro-Oeste & 18.902 & $1.890,2$ & $14.989 .167,0$ & 193,26 \\
\hline Total & $\mathbf{2 7 1 . 1 4 2}$ & $\mathbf{2 7 . 1 1 4 , 2}$ & $\mathbf{1 9 9 . 9 2 0 . 9 9 0 , 2}$ & 126,10 \\
\hline
\end{tabular}

Legenda: *Teste de tendência do $x^{2}(p<0,05)$. Fonte: Ministério da Saúde - Sistema de Informações Hospitalares do SUS (SIH/SUS), 2019.

Em relação aos gastos hospitalares, observa-se proporção direta com o número de procedimentos cirúrgicos. O gasto médio geral foi de 352,59 reais. O Sudeste teve o maior gasto hospitalar com profissionais e não-especificado, com valor médio de 366,12 reais por cirurgia. O Centro-oeste teve o menor valor médio gasto, com 319,33 reais, conforme consta na Tabela 3.

Tabela 3 - Valor, em reais, dos serviços hospitalares decorrentes da realização da vasectomia distribuídos por ano e por região brasileira, entre 2009 a 2018.

\begin{tabular}{|c|c|c|c|c|c|}
\hline Regiões & $\begin{array}{l}\text { Número de } \\
\text { Vasectomias }\end{array}$ & $\begin{array}{l}\text { Gasto } \\
\text { Hospitalar }\end{array}$ & $\begin{array}{l}\text { Gasto com } \\
\text { Profissionais }\end{array}$ & $\begin{array}{l}\text { Gasto não- } \\
\text { Especificado }\end{array}$ & Gasto Médio* \\
\hline Norte & 5.914 & $804.940,07$ & $1.040 .057,06$ & $45.842,59$ & 319,33 \\
\hline Nordeste & 38.286 & $5.307 .637,64$ & $6.892 .101,74$ & $334.829,95$ & 327,31 \\
\hline Sudeste & 152.715 & $22.148 .355,16$ & $27.340 .160,25$ & $6.469 .000,62$ & 366,12 \\
\hline Sul & 55.325 & $7.946 .292,48$ & $9.216 .730,38$ & $2.098 .907,37$ & 347,75 \\
\hline Centro-Oeste & 18.902 & $2.695 .174,38$ & $3.304 .960,59$ & $35.703,58$ & 319,11 \\
\hline Total & 271.142 & $38.902 .399,73$ & 47.794.010,02 & $8.984 .284,11$ & 352,59 \\
\hline
\end{tabular}

Legenda: *Teste de tendência do $x^{2}(p<0,05)$. Fonte: Ministério da Saúde - Sistema de Informações Hospitalares do SUS (SIH/SUS), 2019.

Foram notificados 2 óbitos no estado de Minas Gerais em 2008 e 2011 relacionados ao procedimento. De acordo com a figura 1, é possível observar a distribuição total da cirurgia de vasectomia por estado. Nota-se que São Paulo, Santa Catarina, Paraná, Bahia, Minas Gerais e Rio Grande do Sul são os estados com os maiores números de vasectomias realizadas no período estudado, variando de 10 mil a pouco mais de 124 mil. Já o Pará, Piauí, Maranhão, Acre, Alagoas e Amapá são os estados que apresentam os menores números, com somatória desses estados equivalendo a menos de 1 mil, como aparece na Figura 1. 
Figura 1 - Distribuição do número total de vasectomias por estado realizadas no período de 2009 a 2018.

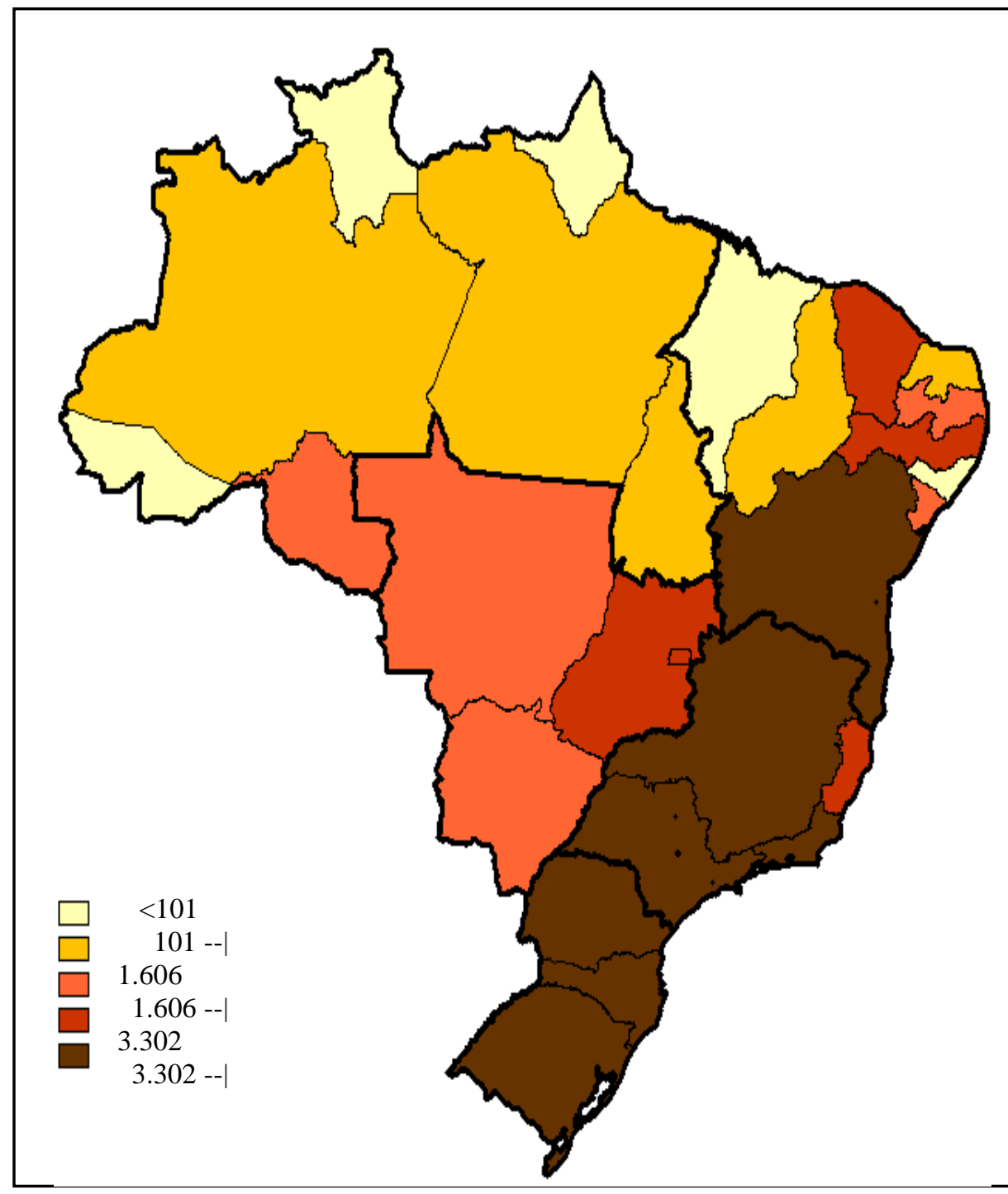

\begin{tabular}{|c|c|}
\hline Estados & Número \\
\hline São Paulo & 120.642 \\
\hline Santa Catarina & 20.061 \\
\hline Paraná & 19.950 \\
\hline Bahia & 16.421 \\
\hline Minas Gerais & 15.640 \\
\hline Rio Grande S & 15.314 \\
\hline Rio de Janeiro & 12.987 \\
\hline Pernambuco & 10.917 \\
\hline Distrito Federal & 6.988 \\
\hline Goiás & 6.876 \\
\hline Ceará & 4.247 \\
\hline Espírito Santo & 3.446 \\
\hline Mato Grosso & 3.310 \\
\hline Sergipe & 3.012 \\
\hline Rondônia & 2.939 \\
\hline Paraíba & 2.757 \\
\hline Mato Grosso S & 1.728 \\
\hline Amazonas & 1.596 \\
\hline Tocantins & 785 \\
\hline Rio Grande N & 673 \\
\hline Pará & 542 \\
\hline Piauí & 130 \\
\hline Maranhão & 103 \\
\hline Acre & 44 \\
\hline Alagoas & 26 \\
\hline Amapá & 8 \\
\hline Total & $\mathbf{2 7 1 . 1 4 2}$ \\
\hline
\end{tabular}

Fonte: Protocolo de pesquisa. Mapa feito através dos dados Sistema de Informações Hospitalares (SIH/SUS), 2019.

\section{DISCUSSÃO}

A vasectomia é considerada um dos métodos contraceptivos mais efetivos e bastante utilizada em países de primeiro mundo, ainda apresenta baixa prevalência em países em desenvolvimento, como o Brasil, país de grande extensão territorial e lugar onde os serviços de saúde ainda não possuem plena resolutividade, principalmente, no que tange a saúde reprodutiva masculina (EISENBERG ML et al., 2009; KAVANAUGH ML e ANDERSON RM, 2013).

Isso ocorre porque o Programa de Planejamento Familiar, desde sua implantação no Brasil, foi voltado às mulheres, tendo em vista que, de acordo com a história, a responsabilidade pelo cuidado com a reprodução e os métodos contraceptivos disponibilizados pelo SUS foram delegados somente às mulheres, mesmo que o Programa fosse voltado tanto ao homem, quanto à mulher. Entretanto, o que se verifica, na maioria das vezes, é a indicação do homem à vasectomia apenas quando a mulher possui contraindicação de utilizar outros métodos de contraceptivos.

Apenas recentemente que um número cada vez maior de homens tem demonstrado maior interesse e senso de responsabilidade em relação aos métodos de contracepção, especialmente em países 
desenvolvidos, independente da condição de saúde do cônjuge (KAVANAUGH ML e ANDERSON RM, 2013; CHAILEE M e MICHELLE MI, 2015; TEMACH AJ, FEKADU GA, ACHAMYELEH AA, 2017).

No Brasil, por outro lado, ainda é recente a participação masculina ativa na opção pelos métodos contraceptivos, sobretudo em regiões mais longínquas das capitais metropolitanas, onde 0 acesso a educação e aos serviços de saúde não estão disponíveis levando a falta de informação e dificuldade de acesso à população (KAVANAUGH ML e ANDERSON RM, 2013).

Apesar disso, neste estudo, observa-se significativo crescimento numérico deste procedimento no SUS, em cerca de 40,5\% no número total. Esse crescimento já foi sugerido por meio de uma pesquisa indireta ao mostrar o aumento da prevalência de $0,8 \%$ de parceiros vasectomizados para 5,1\%, entre 1986 e 2006 (BRASIL, 2006; PINTO et al., 2019).

Apesar do interesse cada vez maior pela vasectomia nas grandes metrópoles, poucos estudos epidemiológicos têm abordado tal temática no Brasil, um país continental e com diferenças entre as 5 grandes regiões (PASQUALOTTO FF et al., 2003; CHAILEE M e MICHELLE MI, 2015; CRISTOPHER EA e MOSHE $W, 2009)$.

O crescimento expressivo na região Norte e Nordeste do país sugere a implementação e início de oferta desses serviços em áreas que antes não existiam, bem como começo da inclusão do homem nos serviços de planejamento familiar. Similar ao que aconteceu no estado de São Paulo que inicialmente o crescimento se deu em torno das grandes metrópoles e, posteriormente, para as regiões mais distantes (BRASIL, 2008; MARCHI NM, et al., 2003; PINTO LOAD, et al., 2019).

Esse crescimento ocorre também por conta de ações de educação em saúde e compartilhamento de informações durante a realização de sala de espera ou nos ambulatórios de planejamento familiar e centros de referência, focando nos direitos sexuais e reprodutivos, sobre anticoncepção e cuidados pré e pósoperatórios relacionados tanto à vasectomia quanto a laqueadura tubária, seus riscos e benefícios.

Tal prática, exercida por profissionais de saúde comprometidos com a melhoria do serviço, desmistifica e dá mais segurança ao paciente sobre a realização da vasectomia para o homem e sua companheira permitindo com que a família opte pelo melhor para o casal (KAVANAUGH ML e ANDERSON RM, 2013).

Este aumento também pode ter relação com a nova caracterização de família e seus múltiplos constituintes relacionado às diversas questões culturais (MARCHI NM et al., 2003). O que se percebe atualmente, é que a mulher está mais ativa em escolher, ou melhor, deixar de escolher o método contraceptivo para si, com menor risco e com melhor recuperação pós-operatória, e o homem, por outro lado, está mais receptivo e colaborativo à esterilização por meio da vasectomia (KAVANAUGH ML e ANDERSON RM, 2013 CRISTOPHER EA e MOSHE W, 2009; SHARMA V et al., 2013).

Ou seja, é consequência do maior senso de responsabilidade do homem em relação à contracepção, preocupação com o valor econômico gasto na criação e educação dos filhos, relatos de experiência bem sucedida de outros casais que realizaram o procedimento, impedimento da mulher que realizar a laqueadura tubária, relativa simplicidade no procedimento em relação à esterilização feminina e, especialmente, o início da oferta da vasectomia no Sistema único de Saúde (CHAILEE M e MICHELLE MI, 2015; CRISTOPHER EA e MOSHE W, 2009; SHARMA V et al., 2013; TEMACH AJ, FEKADU GA, ACHAMYELEH AA, 2017).

Nesse quesito, observa-se heterogeneidade na distribuição do número de vasectomias pelo SUS, principalmente ao comparar o Norte, responsável por 5.914 cirurgias e taxa de 34,93 a cada 100 mil habitantes, com o Sudeste, com 152.715 procedimentos e taxa de 181,77 a cada 100 mil habitantes. Isso é relacionado por conta das disparidades econômicas, sociais e de educação que são observadas em inúmeros estudos. O Sudeste por ser mais desenvolvido e, consequentemente, possuir maior rede de serviços de saúde que oferecem a cirurgia, apresenta maior o número (BRASIL, 2008).

Diferentemente da região Norte, cuja dificuldade no acesso à saúde e ao planejamento familiar dificultam o devido encaminhamento para a realização da vasectomia pelo SUS (CHAILEE M e MICHELLE MI, 2015; MOSHER WD et al., 2004; OSIS MJD et al., 2009; COSTA CC, 2016). 
Ao estratificar por estado, nota-se acentuação das diferenças interestaduais, por exemplo, São Paulo teve cerca de 120 mil cirurgias entre os anos de 2008 a 2019, ao passo que o estado do Amapá, apenas 8 no mesmo período. Isso se dá por conta da menor concentração de estabelecimentos de saúde aptos a realizar os procedimentos nas regiões mais longínquas das capitais do Brasil, como a região Norte e Nordeste (PASQUALOTTO FF et al., 2003; COSTA CC, 2016).

Isso mostra a necessidade da implementação de políticas públicas que ofereçam os serviços de planejamento familiar incluindo o homem no processo, independentemente do local de residência, respeitando os princípios da universalidade e desconcentração da rede hospitalar para do centro para as regiões mais interioranas (EISENBERG ML et al., 2009; COSTA CC, 2016).

O crescimento do número dos procedimentos também não foi homogêneo comparando o ano de 2009 ao ano de 2018. O Sudeste diminuiu o número em $11 \%$, diferindo do Norte e Nordeste com crescimento de $876 \%$ e 129\%, respectivamente. Essa regressão se dá porque o Sudeste possui as maiores regiões metropolitanas do país e, desde os anos 80 (BRASIL, 1987), já vem apresentando altos números de prevalência em relação à vasectomia. Ou seja, o maior desenvolvimento econômico e educacional, juntamente com a diminuição da taxa de natalidade (EISENBERG ML et al., 2009; OSTROWSKI KA et al., 2018), corroborou para que mais pessoas pudessem arcar com os custos da cirurgia em uma rede particular ${ }^{16}$, diminuindo a demanda na rede pública (MARCHI NM et al., 2003; COSTA CC, 2016).

Em estudo realizado em São Paulo, em 2016, todos os pacientes que optaram pela esterilização cirúrgica se relataram estar satisfeitos por terem escolhido a vasectomia descrevendo vários aspectos positivos que os influenciaram para tal escolha, como: a melhora no relacionamento sexual o cônjuge, especialmente pela superação do medo de uma gravidez não planejada, assim como menor preocupação com o uso de métodos anticoncepcionais hormonais que potencialmente podiam trazer riscos graves à saúde feminina (MARCHI MN et al., 2011; CHAILEE M e MICHELLE MI, 2015; COSTA CC, 2016).

Em pesquisa realizada em 2016, afirma que a maioria dos casais que optaram pela vasectomia, utilizavam mais de um tipo de método isolado ou combinado antes de sua realização. Dentre os métodos, destacam-se: os métodos de barreira, consistindo em obstáculos mecânicos e/ou químicos contra à entrada dos gametas masculinos no canal cervical.

Os métodos de barreira distribuídos no Brasil são os preservativos masculinos e, diafragma; Há também os métodos hormonais, que consistem em hormônios sintéticos similares aos endógenos produzidos pelos ovários, distribuídos em pílulas de anticoncepcional oral e injetável em suas formas isoladas e combinadas de estrógeno e progesterona, porém com uma série de contraindicações e, muitas vezes, com efeitos adversos que levam muitas mulheres em vida sexual ativa a não usá-los (MOSHER WD et al., 2004; CHAILEE M e MICHELLE MI, 2015; EISENBERG ML et al., 2009; OSTROWSKI KA et al., 2018).

A escassez de recursos humanos e físicos torna-se um fator que colabora para dificultar o acesso à vasectomia e aumenta a fila de espera, estimulando os homens a desistirem desse método contraceptivo e influenciando suas companheiras a optar pela laqueadura tubária, sendo esta mais onerosa ao SUS, oferecendo maior risco à saúde feminina, porém, com menos demorada que a esterilização masculina indicando que a demanda recebida nos serviços públicos de saúde não conseguem resolução, implicando demora maior para obter a vasectomia do que a laqueadura. Isso inviabiliza a livre escolha e dos métodos anticoncepcionais e contribui para a esterilização tubária (OSIS MJD et al., 2003; OSSIS MJD et al., 2006; CHAILEE M e MICHELLE MI, 2015)

Por fim, oferecer a opção pela vasectomia a casais durante a rotina assistencial é fundamental para um cuidado mais voltado à saúde dos homens, especialmente, para inseri-los no grupo que necessita de atenção à saúde reprodutiva (EISENBERG ML et al., 2009; OSTROWSKI KA et al., 2018).

A inserção do homem no bojo da saúde reprodutiva está intrinsecamente relacionada aos princípios do SUS, especialmente, nas diretrizes que norteiam a integralidade para todas as pessoas atendidas. É importante destacar que não é simplesmente a troca da contracepção definitiva feminina pela masculina, mas de permitir aos homens também a escolha livre e esclarecida de um método eficaz, com menos riscos quando 
comparado à esterilização feminina e com rápida recuperação pós-operatória, menos complicações e dias de internação reduzidas ou inexistentes (EISENBERG ML et al., 2009; COSTA CC, 2016).

Como viés de estudo, destaca-se a fragilidade de dados não preenchidos completamente no sistema de informação do Sistema Único de Saúde. Porém, ainda é possível calcular tendências e taxas de frequência planejando ações para melhorar indicadores de saúde em determinadas áreas. Além disso, serve para mostrar as regiões onde a notificação de procedimentos cirúrgicos, como a vasectomia, precisa ser mais bem trabalhada por meio de treinamento dos profissionais para o correto preenchimento das fichas de notificação (SANTOS DR et al., 2017).

Este estudo é o primeiro no Brasil a abordar o perfil epidemiológico especificamente das vasectomias pelo Sistema Único de Saúde e, apesar dos possíveis vieses, servirá para subsidiar novas pesquisas no campo do planejamento familiar e da saúde reprodutiva masculina.

\section{CONCLUSÃO}

Apesar das diferenças observadas entre as regiões feitas por este estudo, pode-se supor que está havendo maior aceitação e procura da realização de vasectomia no Brasil mediante aumento do número de procedimentos notificados no Sistema Único de Saúde. Nota-se desproporção na distribuição na região Norte e Nordeste em comparação às demais regiões, devido à dificuldade de acesso aos serviços de saúde. Necessita-se, portanto, de ampliação das redes públicas de saúde que ofereçam o serviço aos homens, sobretudo, nas regiões onde não há serviços de planejamento familiar masculino, respeitando, dessa maneira a integralidade do cuidado. Este estudo contribui para a melhor compreensão da distribuição da realização de vasectomia no Brasil na última década, possibilitando a elaboração de estratégias pelos gestores de saúde para melhoras o acesso ao serviço e implementar novos serviços em áreas onde a concentração da vasectomia é baixa. Além disso, esse trabalho mostra o quantitativo de procedimentos cirúrgicos realizados pelo SUS para profissionais urologistas e comunidade acadêmica e sociedade.

\section{REFERÊNCIAS}

1. BRASIL. Ministério da Saúde. Pesquisa nacional sobre saúde materno-infantil e planejamento familiar - PNSMIPF Brasil 1986. Rio de janeiro: bemfam,1987.

2. BRASIL. Ministério da Saúde. Pesquisa Nacional de Demografia e Saúde da Criança e da Mulher, 2006. Brasília: Ministério da Saúde, 2008.

3. CHAILEE M, MICHELLE MI. Sterilization: a review and update. Obstet Gynecol Clin N Am. 2015.

4. CHRISTOPHER EA, MOSHE W. Risks and Complications of Vasectomy. Urol Clin N Am 36 (2009) 331-336.

5. EISENBERG ML, LIPSHULTZ LI. Estimating the number of vasectomies performed annually in the United States: data from the National Survey of Family Growth. J Urol, 2010. 184(5): p. 2068-72.

6. EISENBERG ML, et al. Racial differences in vasectomy utilization in the United States: data from the national survey of family growth. Urology, 2009. 74(5): p. 1020-4.

7. KAVANAUGH ML e ANDERSON RM. Contraception and Beyond: The Health Benefits of Services Provided at Family Planning Centers, New York: Guttmacher Institute, 2013.

8. MARCHI NM, et al. Opção pela vasectomia e relações de gênero. Cad. Saúde Pública. 2003, vol.19, n.4, pp.10171027.

9. VIEIRA EM. A esterilização de mulheres de baixa renda em região metropolitana do sudoeste do Brasil e fatores ligados à sua prevalência. Rev Saúde Pública 1994; 28:440-8.

10. MOSHER WD, et al. Use of contraception and use of family planning services in the United States: 1982-2002. Adv Data 2004; Dec (350):1-36.

11. OSIS MJD, et al. Atendimento à demanda pela esterilização cirúrgica na Região Metropolitana de Campinas, São Paulo, Brasil: percepção de gestores e profissionais dos serviços públicos de saúde. Cad. Saúde Pública, Rio de Janeiro, 25(3):625-634, mar, 2009.

12. OSIS MJD, et al. Fertility and reproductive history of sterilized and non-sterilized women in Campinas, São Pau- lo, Brazil. Cad Saúde Pública 2003; 19:1399-404.

13. OSTROWSKI KA, et al. Evaluation of Vasectomy Trends in the United States. Urology, Volume 118, 76 - 79. 2018.

14. SCAFURI G. Aspectos médicos legais da vasectomia sob a ópti- ca penal. Urologia contemporânea, Jan/Mar 2002: 32-37p. Senado Federal. Lei no. 9.263, de 12 de janeiro de 1996. Brasília: Diário Oficial da União 1996; 15 jan.

15. PASQUALOTTO FF, et al. Trends in male contraception. Rev. Hosp. Clín. Fac. Med. S. Paulo 58(5):275-283, 2003.

16. PINTO LOAD, et al. Portable model for vasectomy reversal training. Int Braz J Urol. 2019 Sep-Oct;45(5):1013-1019. 
17. Costa AM, Guilhem D, Silver LD. Planejamento familiar: a autonomia das mulheres sob questão. Rev Bras Saúde Matern Infant 2006; 6:75-84.

18. ROMERO PP, et al. La vasectomía: estudio de 300 intervenciones. Revisión de la literatura nacional y de sus complicaciones. Actas Urológicas Españolas, 2004. 28(3), 175-214.

19. SHARMA V, et al. Vasectomy demographics and postvasectomy desire for future children: results from a contemporary national survey. Fertil Steril, 2013. 99(7): p. 1880-5.

20. TEMACH AJ, FEKADU GA, ACHAMYELEH AA. Educational status as determinant of men's knowledge about vasectomy in Dangila town administration, Amhara region, Northwest. Reproductive Health (2017) 14:54.

21. Santos DR, et al. Mortalidade materna na população indígena e não indígena no Pará: contribuição para a vigilância de óbitos. Esc Anna Nery. 2017;21(4):1-9. http://dx.doi.org/10.1590/2177-9465-ean-2017-0161.

22. Costa CC. A Esterilização Masculina: perfil e percepções de homens que optaram pela vasectomia. O Social em Questão - Ano XIX - nำ 36 - 2016. 